\title{
Methods to Determine Optimal Washout Position for Single and Multi-Occupant Motion Simulator
}

\author{
Kong Xiang-Tong, Zhu Yuan-Chang, Di Yan-Qiang, Cui Hao-Hao \\ Department of Electrics and Optics Engineering, Mechanical Engineering College, 050003 \\ Shijiazhuang, China \\ Emails: kongxiangtong28@163.com584797300@qq.com 2399886228@qq.com cuisim812@ sina.com
}

\begin{abstract}
The washout position has great effects on the perception fidelity and the movement of the motion simulator. In this paper, the main square error of sensation between simulator and vehicle is determined, and the fidelity of the occupant and the platform motion performance as a basis to build a model for a single occupant optimal washout position measurement. Then, considering the features of the multioccupant motion simulator, a concept of the task's master-crew is proposed, the multi-occupant optimal washout position measurement model is built. Then an antiaircraft simulator for three occupants is taken as an example of a multi-occupant simulator and the optimal washout position measurement model is studied. Considering that the basic PSO algorithm has shortcomings such as easy aging and falling into local extreme value, a modified algorithm is designed called Adaptive Chaos PSO algorithm and applied to the resolution of the models. The results showed that the optimal position can make the simulator trainer have a more realistic sensation and maintain the safety of the platform, and that the Adaptive chaos PSO algorithm can effectively solve the shortcomings of PSO and is very useful in the resolution of the model.
\end{abstract}

Keywords: Motion Cueing algorithm, multi-occupant, washout position, ACPSO, simulator.

\section{Introduction}

The Stewart platform has the advantages of great rigidity, high-force-weight ratio, and a mobility of six degrees of freedom of movement. It has been widely used in motion simulator systems, such as flight simulators and vehicle simulators [1]. The goal of the motion simulator is the reproduction of dynamics such as accelerations and rotations. This is an intricate task because the motion hardware displacement is subject to limitations. Therefore washout algorithms (also called motion cueing algorithm) are required that translate the vehicle motion into valid simulator 
displacements [1]. Several different types of washout algorithms exist, including the classical washout algorithm, the adaptive washout algorithm and the optimal washout algorithm. The classical washout algorithm is the most common approach and has been widely used $[2,3]$.

The washout position is a key factor for the effects of a motion simulator and is essential for the fidelity of the motion cueing. In the existing literature, there is not much research on how to choose the optimal washout position. For the single crew motion simulator, the washout position is generally considered as the centroid of platform or the crew's vestibular place $[4,5]$, while with the double crew flight simulator is considered as the midpoint of the pilots' head [6]. The reasons to choose these positions are some simple comparisons of several possible points that lack depth analysis and clear criteria. In addition, a simulator with three or more crew occupants, such as its own anti-aircraft gun, has not yet been specifically researched. For these multi-occupant systems, the occupant distribution in different positions and sensations may result in a larger variations, so the washout positions must be more accurate and rational.

\section{Washout filter and washout position}

The washout filter is a necessary linking part between the movements of the actual vehicle and the simulator, and the washout position is an important reference point between the parameters of actual movement and the washout algorithm input, having a great influence on the simulator [7].

\subsection{Classical washout filter}

Many related research projects on the washout filter have been presented in the past decades. The classical washout filter is one of the most commonly used, which is composed of a linear acceleration high-pass filter, a tilt coordinate low-pass filter and an angular velocity high-pass filter $[8,9]$. The input signals are specific force and angular velocity in the washout position, and the output signal is the platform's pose. The flow chart of a washout filter is shown in Fig. 1, and the details are discussed below.

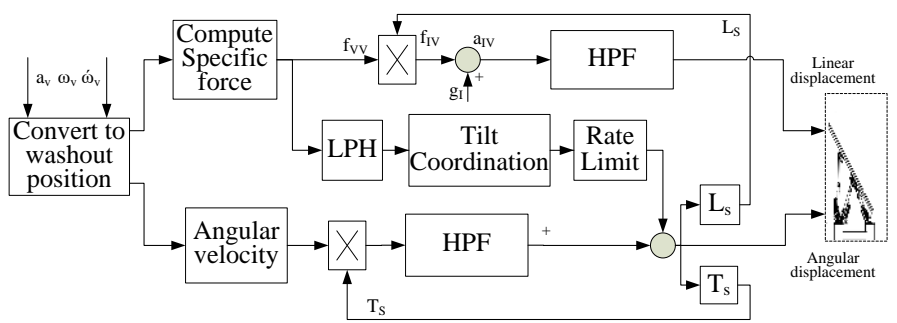

Fig. 1. Classical washout filter flow chart

The specific force $f_{x}, f_{y}$ and $f_{z}$ are computed by subtracting $g$ from the given linear acceleration $a$. The high frequency components of the specific force are obtained by the following 3rd-order HPF: 


$$
H_{a_{-} \mathrm{HP}}=\frac{s^{2}}{s^{2}+2 \varepsilon_{a_{-} \mathrm{HP}} \omega_{a_{-} \mathrm{HP}}+\omega_{a_{-} \mathrm{HP}}^{2}} \cdot \frac{s}{s+\omega_{b}} .
$$

This HPF filters out low-frequency motion signals which tend to cause large linear displacement that causes the platform to exceed its limits. The low frequency components of the specific force are obtained by the following 2nd-order LPF:

$$
H_{\mathrm{LP}}=\frac{\omega_{\mathrm{LP}}^{2}}{s^{2}+2 \varepsilon_{\mathrm{LP}} \omega_{\mathrm{LP}} s+\omega_{\mathrm{LP}}^{2}} .
$$

The output signals are set after LPF is realized by tilting the platform along the $\mathrm{x}$ and $\mathrm{y}$ axes, causing one to perceive it as a real specific force when the body tilts. This is called tilt coordination. In [16], the tilt rate was limited to $-5^{\circ}$ per $1 \mathrm{~s}$ preventing the person from perceiving the movement. The pitch angle and roll angle are set to

$$
\theta_{q}=f_{\mathrm{LS} x} / g, \quad \varphi_{p}=-f_{\mathrm{LS} y} / g .
$$

The high-frequency components of the angular velocity are obtained by the following 2nd-order HPF and then integrated to set the angular displacement of the platform:

$$
H_{\beta_{-} \mathrm{HP}}=\frac{s^{2}}{s^{2}+2 \varepsilon_{\beta_{-} \mathrm{HP}} \omega_{\beta_{-} \mathrm{HP}}+\omega_{\beta_{-} \mathrm{HP}}^{2}} .
$$

\subsection{Influences of washout position}

The motion parameters of the centre of gravity of the vehicle usually cannot be used directly and need to be converted to the washout position to balance the platform movement and the human sensation. According to the theory of rigid body kinematics, the linear acceleration and angular velocity of the washout position are:

$$
\begin{gathered}
\vec{a}_{\mathrm{w}}=\vec{a}_{\mathrm{CG}}^{V}+\overrightarrow{\dot{\omega}}_{V} \times \vec{r}_{\mathrm{w}}+\vec{\omega}_{V} \times\left(\vec{\omega}_{V} \times \vec{r}_{\mathrm{w}}\right), \\
\vec{\omega}_{\mathrm{w}}=\vec{\omega}_{V},
\end{gathered}
$$

where $\vec{r}_{\mathrm{w}}$ denotes the relative position vector between the washout position and CG (Centre of Gravity) of the vehicle; $\vec{a}$ denotes linear acceleration and $\vec{\omega}$ denotes angular velocity; the subscript CG denotes the centre of gravity of the vehicle and $w$ denotes the washout position.

As can be seen, the washout position influences the input of the washout filter directly, and it also influences the displacement of the platform and the perception of the occupants in the simulator. So the washout position plays an important role in the simulator and needs to be determined precisely.

\section{Single occupant optimal washout position model}

The washout position is of great significance to the simulator, which is closely related to the occupants' sensations and platform displacement. References $[4,10]$ show the chosen method and indicate that it can be chosen at any position on the 
simulator and most often chosen at the drivers' vestibular center and the mass center of the motion platform. Reference [4] compares the washout effects of the commonly used washout positions and illustrates that mass center of the upper platform is the best position. However, the research above only focused on several specific positions without considering other possible positions. At the same time, the effect evaluation is mostly qualitative and macro rather than adhering to strict quantitative standards. Hence it is essential to make further research on the method of washout potion selection.

\subsection{Optimal washout position measurement criteria of single occupant simulator}

Optimal washout position measurement criteria of the single occupant simulator must comprehensively take into account both the platform motion limits and occupants' perception fidelity. The platform movement capacity means to get the platform to move as extensively as it can, according to the conditions within its actuators' elongation. The actuator's elongation $l_{i}, i=1, \ldots, 6$, can be calculated with an inverse kinematic model based on platform attitudes [11, 15] (Euler angles $\vec{\beta}_{p}$ and displacement $\vec{S}_{p}$ ).

The fidelity of the occupants' sensation is measured by two criteria; the correlation coefficient $r$ and the MSE (Mean Square Error) $f$ between actual sensation in simulator and sensation of the real vehicle. The correlation coefficient is used to evaluate the trend consistency and the MSE is to evaluate the error [9]. The function of MSE is

$$
f=\int_{0}^{t}\left(f_{z s}-f_{p t}\right)^{2} d t
$$

where $f_{z s}$ and $f_{p t}$ are the perception in the real vehicle and the simulator.

When initial settings are constant, $f$ and $r$ can be seen as the function of $\vec{r}_{w}$ that denotes the vector of the washout position. The smaller the $f$ and the larger the $r$, the higher the simulation fidelity. So, according to the analysis above, the problem of resolving the optimal washout position is a typical Multi-Objective Optimization Problem (MOOP) and the required optimization targets are three:

1) The platform motion should be with less cost, $\min \left(\sum_{i=1}^{6}\left|l_{i}\right|\right)$.

2) Trends in the occupants' sensation variation should be similar, namely the correlation coefficient should be larger, $\max (r)$.

3) The MSE of the occupants' perception between the real vehicle and the simulator should be a minimum, thus, $\min (f)$.

The restricted condition is the platform movement within its limits, that is to say the actuators' extension within limits:

$$
\text { s.t. }\left\{\begin{array}{l}
l_{i}-l_{i_{-} \min } \geq 0, \\
l_{i}-l_{i_{-} \max } \leq 0,
\end{array} \quad i=1,2, \ldots, 6 .\right.
$$


For the problem with restricted conditions, the general method is to convert it to an unconstrained situation with a penalty function method, so define the function:

$$
\left\{\begin{array}{c}
g_{i}(\vec{x})=l_{\min }-l_{i}(\vec{x}) \leq 0, \\
g_{i+6}(\vec{x})=l_{i}(\vec{x})-l_{\max } \leq 0,
\end{array} i=1,2, \ldots, 6 .\right.
$$

Above all, the fitness value function with penalty function with SMUT method is

$$
F(\vec{x})=\sum_{i=1}^{6}\left|l_{i}(\vec{x})\right|+\frac{1}{r(\vec{x})}+f(\vec{x})+m \sum_{k=1}^{12}\left(g_{k}(\vec{x})\right)^{-1},
$$

where $m$ denotes the penalty factor, $\vec{x}=\left(x_{1}, x_{2}, x_{3}\right)$ denotes the vector of the parameters to be resolved. The best solution is the vector $\vec{x}$ that makes $F$ the minimum value.

\subsection{Steps of the single occupant mode algorithm}

The intelligence optimization algorithms are commonly used to solve the multiobjective optimization problem, such as the Particle Swarm Optimization (PSO) algorithm. Aiming at this problem, the schematic diagram is shown in Fig. 2, and the concrete steps are seven.

Step 1. Calculate the vehicle dynamic parameters at certain definite conditions, which are linear acceleration $\vec{a}_{\mathrm{CG}}^{V}$, angular velocity $\vec{\omega}_{V}$, and angular acceleration $\overrightarrow{\dot{\omega}}_{V}$. Then save them in the memory.

Step 2. Calculate the sensation in the real vehicle. First, calculate the occupants' motion parameters $\vec{\omega}_{\mathrm{h}}$ and $\vec{a}_{\mathrm{h}}$ in the head position based on the relative position between occupants' position and vehicle mass center. Next, transform $\vec{a}_{\mathrm{h}}$ to the specific force $\overrightarrow{\widehat{f}}_{\mathrm{h}}$ in inertial frame. Finally, determine the motion sensation $\overrightarrow{\hat{\omega}}_{\mathrm{h}}$ and $\overrightarrow{\widehat{f}}_{\mathrm{h}}$ by letting $\vec{\omega}_{\mathrm{h}}$ and $\overrightarrow{\widehat{f}}_{\mathrm{h}}$ through the vestibular model.

Step 3. Based on the position vector between the washout position that the optimization algorithm generated and the vehicle mass center, combined with the vehicle movement parameters stored in Step 1, calculate motion parameters $\vec{\omega}_{\mathrm{w}}$ and $\vec{a}_{\mathrm{w}}$ at the washout position.

Step 4. Calculate the platform motion parameters and the actuator elongation. The platform position and orientation can be acquired by letting motion parameters in the washout position, noted as $\vec{\beta}_{\mathrm{p}}$ and $\vec{S}_{\mathrm{p}}$, through the washout filter, then further determine the actuator elongation $\vec{l}_{\mathrm{p}}$ by the inverse kinematic model. 


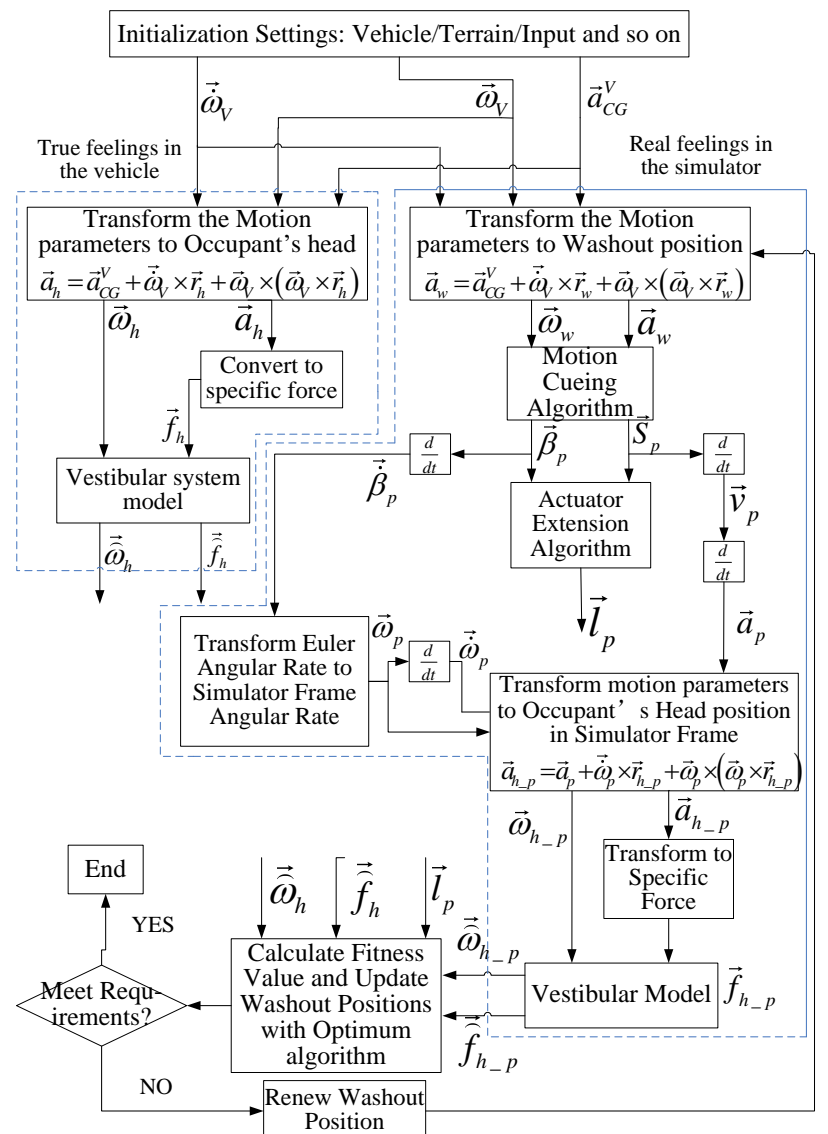

Fig. 2. Diagram of single occupant optimal washout position algorithm

Step 5. Calculate the occupants' sensation in the simulator. According to angular velocity $\vec{\omega}_{\mathrm{p}}$, angular acceleration $\overrightarrow{\dot{\omega}}_{\mathrm{p}}$, and linear acceleration $\vec{a}_{\mathrm{p}}$ of the platform acquired by derivative of $\vec{\beta}_{\mathrm{p}}$ and $\vec{S}_{\mathrm{p}}$, combined with the relative position vector $\vec{r}_{\mathrm{h}_{\mathrm{p}}}$ between simulator occupants and upper-platform center and human vestibular model, we can determine the occupants' simulator sensation $\overrightarrow{\hat{\omega}}_{\mathrm{h} \_\mathrm{p}}$ and $\overrightarrow{\hat{f}}_{\mathrm{h} \_\mathrm{p}}$.

Step 6. Calculate the fitness value of the washout position. According to the parameters obtained from the steps above, which are $\overrightarrow{\hat{\omega}}_{\mathrm{h}}, \overrightarrow{\hat{f}}_{\mathrm{h}}, \overrightarrow{\hat{\omega}}_{\mathrm{h} \_\mathrm{p}}, \overrightarrow{\hat{f}}_{\mathrm{h} \_\mathrm{p}}$ and $\vec{l}_{\mathrm{p}}$, by function (9) we can calculate the fitness value $F$.

Step 7. Determine the optimal washout position. The optimal position vector $\vec{x}_{i}$ is the vector that makes fitness value $F$ the minimum, if the end condition is not met turn to Step 3 and continue the loop until the end term is met and output the optimal value. 


\section{Multi-occupant optimal washout position model}

The single occupant simulator only focuses on one trainer, while the multi-occupant simulator usually accommodates three or more trainers located in different positions, as well as in different tasks. So the way to obtain the optimal washout position for a multi-occupant simulator is different from the single occupant simulator. The multi-trainer mode washout position strategy needs to balance all the trainers' sensations and the platform's motion ability. For these reasons, we need to make further research on the optimal washout position model for a multi-occupant simulator.

\subsection{Optimal washout position measurement criteria of multi-occupant simulator}

Various simulators often have various trainer quantity and distribution styles, so the optimal washout position measurement criteria often have personalized characteristics, and the way to solve the problem can be used as reference for various types of multi occupant simulators. This section considers a Self-Propelled Anti-Aircraft Gun (SPAAG) simulator, which is shown in Fig. 3, as an example to resolve its optimal washout position. The approach can be extended to other types of multi-occupant simulators.

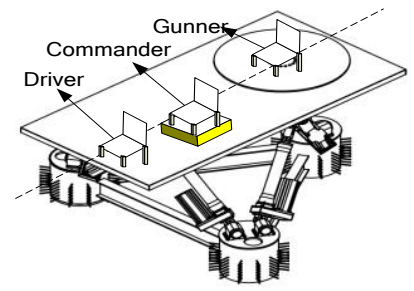

Fig. 3. Diagram of the three occupant anti-aircraft gun simulator

The SPAAG simulator consists of three occupants who are driver, commander and gunner. The commander is responsible for commanding and target research-:The gunner is responsible for tracking and shooting. The driver is responsible for driving. When performing different tasks, one of the crew tends to play a leading role, while the rest of the master crew perform a specific task, such as the driver in the driving task.

The sensations of the trainers in the simulator are different for the distribution difference. To ensure the training's effectiveness, we establish the optimal washout position measurement criteria of the multi-occupant simulator that is dominated by the training task. That is to say, the task's master-crew has relatively high sensation fidelity while others also have some degree of sensation fidelity within the platform motion range. The method is specified by taking the drive task as an example.

The drive task is an important training subject for the SPAAG simulator. The driver is the master-crew, and the commander and gunner are secondary crew members. The measurement criteria must first meet the driver's sensation fidelity and also give consideration to both the commander and gunner. To ensure sensation fidelity of all the trainers, the washout point is set at the common washout position (the center of the upper platform), demonstrated in $[4,10]$ as the general rule. The 
requested measurement criteria under the condition that the commander, gunner and driver are no less than $75 \%$ and $85 \%$ of the sensation respectively, which makes the driver's sensation fidelity the best. So, besides the function (7), the restricted sensation condition also needs to meet:

$$
\text { s.t. } f_{p}(\vec{x}) \leq \frac{f_{p_{-} z x}}{q}, \quad p=1,2,3 .
$$

For this restricted condition, the penalty function is defined as

$$
h_{p}(\vec{x})=f_{p}(\vec{x})-\frac{f_{p_{-} z x}}{q} \leq 0, \quad p=1,2,3 .
$$

where $f_{p}$ and $f_{p_{-} z x}$ represent the MSE and rule MSE of a certain trainer's sensation. When $p$ equals 1 or 2 , it denotes the parameters for the commander and gunner, when the parameter $q$ is 0.75 ; if $p$ equals to 3 , it denotes the parameters for the driver, when the parameter $q$ is 0.85 .

Under the restricted conditions above, besides the single mode optimization target (1), the optimization targets must also meet the conditions below:

1) The correlation coefficient of all occupants' senses should be the maximum, that is

$$
\max \left(\sum_{j=1}^{3} \frac{1}{r_{j}(\vec{x})}\right),
$$

where $r_{j}(\vec{x})$ denotes the correlation coefficient and when subscript $j$ equals to 1 , 2 , and 3 , it signifies the parameters of the commander, gunner, and driver respectively.

2) The MSE of the driver's sensation should be the minimum $-\min \left(f_{j s y}\right)$, where $f_{j s y}$ denotes the MSE of the driver.

Above all, the fitness calculation function constructed with a penalty function is

(13) $F(\vec{x})=\sum_{i=1}^{6}\left|l_{i}(\vec{x})\right|+\sum_{j=1}^{3} \frac{1}{r_{j}(\vec{x})}+f_{j s y}(\vec{x})+m \sum_{k=1}^{12}\left(g_{k}(\vec{x})\right)^{-1}+n \sum_{p=1}^{3}\left(h_{\mathrm{p}}(\vec{x})\right)^{-1}$,

where $m$ and $n$ denote the penalty factor, $\vec{x}$ denotes the vector of the parameters to be solved.

\subsection{Steps of the multi occupant mode algorithm}

The process of the multi occupant mode algorithm is shown in Fig. 4. Most of parameter calculation methods have been discussed above, so we shall not go into further discussion. The main steps are six.

Step 1. Calculate the vehicle dynamic parameters on a certain condition and then save them in the memory.

Step 2. Calculate the sensations of all occupants in the real vehicle based on their position. 
Step 3. Calculate the sensations of all trainers in the simulator and the actuator's elongation based on the trainer's position and the current washout position.

Step 4. Calculate the correlation coefficients and MSE of all occupants.

Step 5. Calculate the fitness value based on the parameters above and the fitness function of each corresponding to the task.

Step 6. Define the optimal washout position according to the ending condition and if not met, turn to Step 3 and continue the loop until it is met.

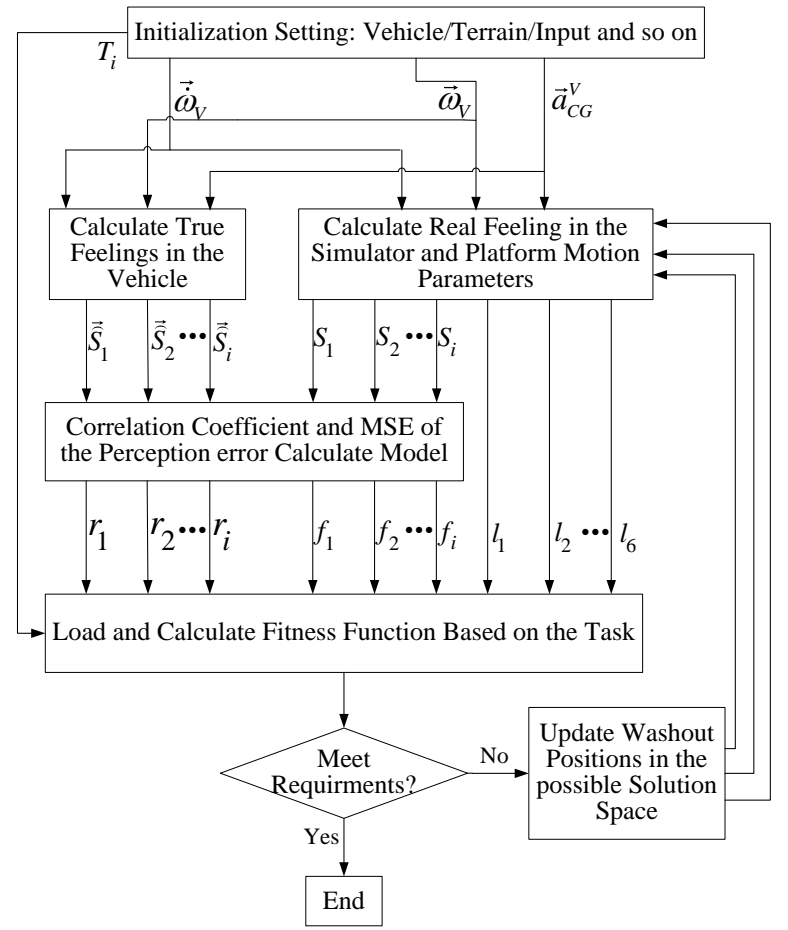

Fig. 4. Diagram of the multi-occupant optimal washout position algorithm

\section{Adaptive chaos PSO algorithm}

PSO algorithm is a kind of intelligence algorithm that simulates swarm intelligence behaviour of nature $[187,198]$. The basic and the improved series algorithms can be used in the optimal washout position algorithm model.

\subsection{Theory of adaptive chaos PSO}

The PSO algorithm takes every potential solution as a particle with fitness value based on the optimization situation in the $D$ dimension space. Each particle has a certain speed that determines its direction and displacement [12]. Particles search for the optimal solution in solution space through iterative, the speed and position of which is updated by their personal extremum and global extremum. For the $i$-th particle, the speed and position update rule [11] are 


$$
\begin{gathered}
v_{i d}^{k+1}=\omega v_{i d}^{k}+c_{1} r_{1}\left(p_{b_{-} i d}^{k}-x_{i d}^{k}\right)+c_{2} r_{2}\left(g_{b_{-} d}^{k}-x_{i d}^{k}\right), \\
x_{i d}^{k+1}=x_{i d}^{k}+v_{i d}^{k+1}, \quad 1 \leq d \leq D,
\end{gathered}
$$

where $x_{i d}^{k}$ and $v_{i d}^{k}$ denote position and speed, $p_{b_{-} i d}^{k}$ and $g_{b_{-} d}^{k}$ denote the personal extremum and local extremum, $\omega$ denotes the inertial factor. The subscript id indicates the $i$-th particle in the $d$-th dimension; the superscript $k$ denotes the $k$-th iteration.

The basic PSO algorithm has a problem of too fast a convergence and being too susceptible to fall into local minimum [16]. To improve the performance of the PSO algorithm, a modified PSO algorithm with inertial factor adjusted adaptively [17] and particles movement following chaos algorithm was proposed which is called Adaptive Chaos PSO (ACPSO) algorithm. The main improvements are two.

1) The inertial factor adjustment strategy is changed from a simple linear update strategy to an adaptive update strategy that is based on individual fitness value and group average fitness value. The specific contents are: assume the particle swarm size is $n$, and the $i$-th particle's fitness value is $f_{i}$, so the average fitness value is $f_{\text {avg }}=\frac{1}{n} \sum_{i=1}^{n} f_{i}$.

The inertial factor adaptive update rules are:

1.1. if $f_{i} \leq f_{\text {avg }}$, the $i$-th particle has a better performance in swarm so the inertial factor can be decreased to accelerate the convergence, the adjusted rule is

$$
\omega=\omega_{\text {min }}+\left(\omega_{\max }-\omega_{\min }\right)\left|\frac{f_{i}-f_{\text {min }}}{f_{\text {avg }}-f_{\min }}\right| .
$$

1.2. If $f_{i}>f_{\text {avg }}$, the $i$-th particle has a less successful performance in the swarm, the inertial factor can be increased to enlarge the search scope, the adjusted rule is

$$
\omega=\omega+\left(\omega_{\max }-\omega_{\min }\right)\left|\frac{f_{i}-f_{\min }}{f_{\text {avg }}-f_{\text {min }}}\right|,
$$

where $\omega_{\max }$ and $\omega_{\min }$ are the maximum and minimum value of inertial factor.

2) To overcome the shortcomings and allow particles to escape from local extremum, the chaos theory $[13,14]$ which has a good random and ergotis character was introduced to the particles' update process.

When updating the particles, $30 \%$ of the best particles are kept to take the chaos search and then the new better particles replace the original old particles. The remaining $70 \%$ of particles are regenerated randomly. By doing this the good characteristics of chaos algorithm can be used to help the particles which are trapped in the local extremum escape and improve the search precision. The chaos system model uses the typical logistic mapping equation and the iterative formula is

$$
z_{\text {iter }+1}=4 z_{\text {iter }}\left(1-z_{\text {iter }}\right), \quad \text { iter }=0,1,2 \ldots
$$




\subsection{Steps of the adaptive chaos PSO algorithm}

According to the discussion above, the steps of the ACPSO algorithm are shown below.

Step 1. Initialize the particle swarm, mainly including the number of particles $n$, dimension of solution space $D$, and the particles' speed $v_{i}$ and position $x_{i}$. Then calculate and initialize personal extremum $p_{\text {best }}$ and global extremum $g_{\text {best }}$.

Step 2. Update particles' speed and position as function (14), also update inertial factor based on function (15) and (16).

Step 3. Calculate each particle's fitness value $F_{i}$.

Step 4. Take the chaos search and update particles based on the second improvement rules.

Step 5. Update personal and global extremum. For personal extremum, if $F_{i}>p_{\text {best }}$, then $p_{\text {best }}=F_{i}$; choose the best $p_{\text {best }}$ as the global extremum $g_{\text {best }}$.

Step 6. According to the end condition, which means the global extremum is adequate or meet the maximum number of iteration, determine whether the algorithm end or not. If we meet the end conditions then output the results or turn to Step 2 and continue the loop.

\section{Results and discussion}

The washout algorithm model is first verified. Then the optimal washout position algorithm is simulated and tested, both single mode and multi-mode, and the result of the optimal washout position are compared with the common washout position to prove the superiority of the optimal washout position algorithm that we propose. Lastly, the performance of the PSO and ACPSO used for resolving the proposed model are simulated and analysed.

\subsection{Results of washout algorithm}

The input signals are: the acceleration along $x$ axis direction which is a unit step signal and lasts $10 \mathrm{~s}$ from 2 up to $12 \mathrm{~s}$, and the signals along other directions are all 0 . The simulation result is shown in Fig. 5.

Fig. $5 \mathrm{a}$ is the platform acceleration along $x$ axis direction, as can be seen in the rising edge of the input signal, the platform has a positive sudden acceleration, and the falling edge is a reverse sudden acceleration, which means the washout algorithm can exactly simulate the sudden movement at the edges of the input signal. Fig. $5 \mathrm{~b}$ is the movement of motion platform showing the platform can timely return to neutral so as to ensure sufficient space for the next movement. Fig. $5 \mathrm{c}$ is the pitch angle of the platform where it can be seen that the platform has a constant pitch motion which means that the washout algorithm can accurately simulate the sustained acceleration with platform tilt-coordinate strategy. In Fig. 5d, the solid line is input specific force and the dotted line is output specific force, and two 
curves can be seen showing a similar trend and size. So the washout algorithm can ensure the trainer's sensation is the same as the input sensation.

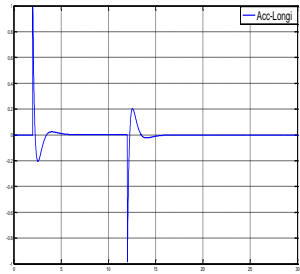

(a)

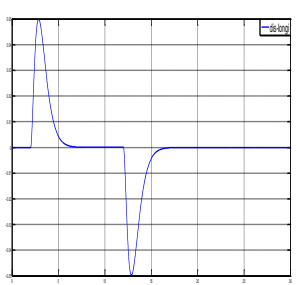

(b)

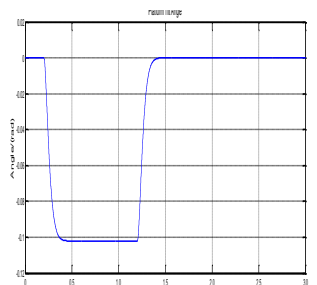

(c)

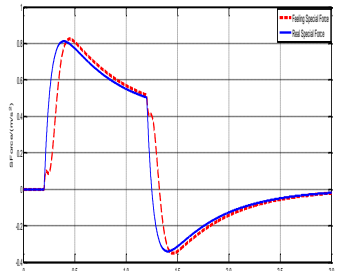

(d)

Fig. 5. Washout algorithm results: Acceleration (a); displacement (b); pitch angle (c); specific force (d)

\subsection{Simulations and analysis of the optimal washout position algorithm}

The object to be simulated is a certain anti-aircraft gun simulator being built. The input signal is shown in Fig. 6 which reveals in Fig. 6a the longitudinal acceleration is a unit step signal lasts $10 \mathrm{~s}$; Fig. $6 \mathrm{~b}$ is pitch angular acceleration signal that shows from 2 up to $5.5 \mathrm{~s}$ and from 12.5 up to $16 \mathrm{~s}$ the value is $2^{\circ}$ per $1 \mathrm{~s}$, from 5.5 up to $12.5 \mathrm{~s}$ is $-2^{\circ}$ per $1 \mathrm{~s}$; inputs signals along other directions are all zero.

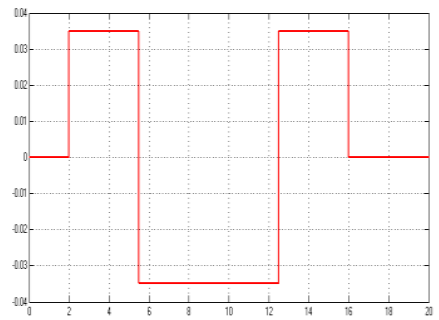

(a)

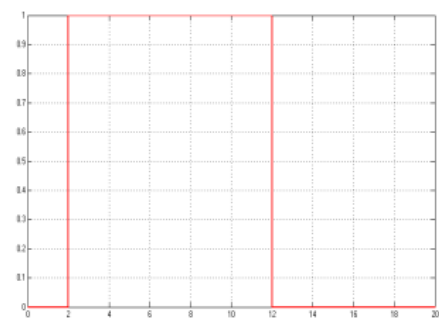

(b)

Fig. 6. The input test signals: Longitudinal acceleration (a); pitch angular (b)

Fig. 7 shows the contrasts of the master occupant's sensations in both single and multi-occupant modes. As is shown in the figure, curve A denotes the output specific force in the common washout position, curve B denotes the output specific force in the real vehicle, curve $\mathrm{C}$ denotes the output specific force in the optimal washout position. Curves B has similar tendency and size with curve $\mathrm{C}$, which means the perception in the two washout positions can both generate the real sensation for the trainer or master trainer no matter single or multi occupant mode. Compared to the common washout position, the optimal washout position can generate a larger signal near the peak, which means the simulation degree is relatively higher, about $5 \%$ higher than the common washout position. 


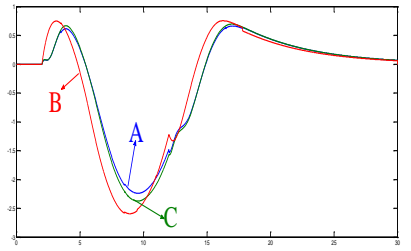

(a)

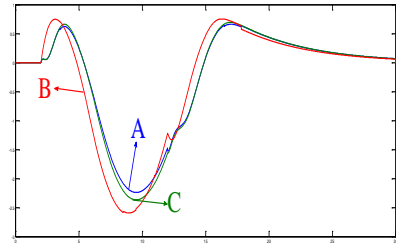

(b)

Fig. 7. Contrast curves of the occupant sensation: Single occupant mode (a); multi occupant mode (b)

Fig. 8 is the contrast curve of the sum of the actuators' elongation in the multi occupant mode, that is $\sum_{i=1}^{6}\left|l_{i}(\mathrm{x})\right|$. The solid line is the curve of the common washout position and the dotted curve is the optimal washout position. From the figure it can be seen that at both washout position, the curves finally get to zero which means the platform returns to the neutral position that meets the actual requirement. Additionally, the dotted line mostly has a larger absolute value than the solid line, which means that in the optimal washout position the platform movement cost is less and maintains the platform movement within its limits. In about 12 seconds the optimal position is larger than the common position, which is approximately the circle zone. This is because the longitudinal acceleration at this time shifts from $1 \mathrm{~m} / \mathrm{s}^{2}$ to 0 , so the platform needs a larger sudden movement to simulate this signal. The larger movement means the simulation of the sudden movement is more realistic. This phenomenon also proves the advantages of the optimal washout position in another side.

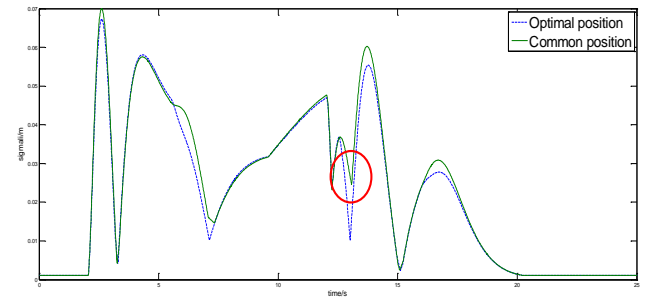

Fig. 8. Contrast of the sum of the actuators' elongation

Fig. 9 shows the contrast curves of the fitness value with a different searching optimization algorithm. The left one is the signal occupant mode and the right one is the multi-occupant mode. The solid line curve indicates the result of the PSO algorithm and the dotted line curve indicates the result of the ACPSO. Experimental results show that the two kinds of optimization algorithms can each find a relatively optimal extremum. The ACPSO algorithm eventually has smaller fitness values relative to the PSO algorithm, which means that it can effectively jump out of the local extremum and further search the global feasible solution space, so the optimization effect is better than the PSO algorithm.

In order to further test the performance of the ACPSO optimization algorithm proposed in this paper, the ACPSO optimization algorithm and the PSO 
optimization algorithm are simulated 10 times each. The maximum iteration for a simulation is 50 and the results are shown in Table 1.

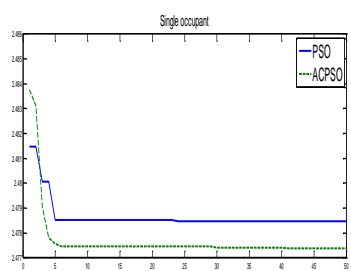

(a)

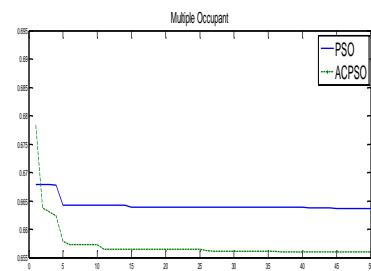

(b)

Fig. 9. Contrast of the fitness value with PSO and ACPSO algorithm: Fitness value of single occupant mode (a); fitness value of multi occupant mode (b)

Table 1 shows the difference between the optimal solution at the 5th simulation and the average optimal solution. This is because the solution accuracy of the PSO algorithm is related to the initial particle position, and can easily fall into local optimal value. The two kinds of results of the ACPSO algorithm are basically identical, which means the effectiveness of the ACPSO algorithm is less affected by the initial particles' position and will not reach the local optimal point. So, compared to the PSO algorithm, the ACPSO algorithm can achieve better global optimal solution with higher precision and has a better performance.

Table 1. Solutions of the PSO and ACPSO

\begin{tabular}{|c|c|c|c|c|}
\hline \multirow{2}{*}{ Mode } & \multicolumn{2}{|c|}{ Optimal solution at 5th simulation } & \multicolumn{2}{c|}{ Average optimal solution } \\
\cline { 2 - 6 } & PSO & ACPSO & PSO & ACPSO \\
\hline $\begin{array}{c}\text { Single } \\
\text { occupant }\end{array}$ & {$\left[\begin{array}{llllll}1.8521 & 0.2633 & 0.5137\end{array}\right]$} & {$\left[\begin{array}{lllll}1.9619 & 0.2633 & 0.5919\end{array}\right]$} & {$\left[\begin{array}{llll}1.8736 & 0.2593 & 0.4851\end{array}\right]$} & {$\left[\begin{array}{llll}1.9619 & 0.2634 & 0.5919\end{array}\right]$} \\
\hline $\begin{array}{c}\text { Multi- } \\
\text { occupant } \\
\text { (drive task) }\end{array}$ & {$\left[\begin{array}{llllll}1.9213 & 0.4644 & 0.4305\end{array}\right]$} & {$\left[\begin{array}{lllll}1.9836 & 0.9641 & 0.3806\end{array}\right]$} & {$\left[\begin{array}{llll}1.8321 & 0.4073 & 0.4327\end{array}\right]$} & {$\left[\begin{array}{llll}1.9836 & 0.9641 & 0.3807\end{array}\right]$} \\
\hline
\end{tabular}

\section{Conclusion}

The washout algorithm can produce sensations, similar to the actual movement for the simulator crew. The washout position is a key factor for motion simulator. In this paper, the single occupant optimal washout position model is first proposed according to the occupant's sensation fidelity and motion platform performance. Then, based on the single occupant mode, the multi-occupant optimal washout position algorithm model is presented based on executed tasks, combined with the measurement criteria with specific tasks. Lastly, combined with the chaos algorithm, an improved adaptive chaos particle swarm optimization algorithm is proposed and applied to the resolution of the proposed models. The simulation result shows the efficiency of the items above. The survey above regarding motion simulator, especially the multi-occupant motion simulator, is of great significance. The proposed ACPSO algorithm can also be used in the study of other multiobjective optimization problems. 
Acknowledgments: We would like to express our sincere appreciation to the anonymous reviewers for their insightful comments, which have greatly helped us in improving the quality of the paper. This work was partially supported by Equipment Pre-research Fund of China under Grant No 9140A04030214JB34058.

\section{References}

1. Lu o, Z.-H., Y.-D. W e i, X.-J., Z Z ou et al. Research on Variable Input Washout Algorithm for Stewart Platform Vehicle Simulator. - Journal of Zhejiang University (Engineering Science), Vol. 47, 2013, No 2, pp. 238-243.

2. Dong, Y., C. Xu, J. Tang et al. Design and Test Research of Washout Filter for 6-DOF Platform. - Journal of Mechanical Engineering, Vol. 46, 2010, No 3, pp. 53-58.

3. Telban, R. J., W. Wu, Cardullo. Motion Cueing Algorithm Development: Initial Investigation and Redesign of the Algorithm. NASA, 2000.

4. W a n g, X. L., L. L i, W. H. Z h a n g. Effect of Washout Locations on the Fidelity of Locomotive Driving Simulator. - Machinery \& Electronics, 2007, No 4, pp. 21-24.

5. W a n g, X. Study on Movement Perception Simulation System for High Speed Train Driving Simulator. Ph.D. Dissertation, Southwest Jiaotong Univ., Chongqing, China, 2009.

6. Z a y chik, K. B., F. M. Cardu 11 o. Nonlinear Motion Cueing Algorithm: Filtering at Pilot Station and Development of the Nonlinear Optimal Filters for Pitch and Roll. State University of New York, Binghamton, New York, NASA/CR-2012-217567, May 2012.

7. Stahl, K., G. Abdulsamad, K. Lei mbach et al. State of the Art and Simulation of Motion Cueing Algorithm for a Six Degree of Freedom Driving Simulator. - In: IEEE 17th International Conference on Intelligent Transportation System (ITSC), Qingdao, 2014, pp. 537-541.

8. W a n g, J.-Z., Y. T an g, X.-L. Zh an g. Proprioceptive Simulation Algorithm for the Driving Simulator System for Special Heavy Vehicles. - Transactions of Beijing Institute of Technology, Vol. 28, 2008, No 8, pp. 670-673.

9. J i a n, G. Research on Fidelity of Motion Cueing System in Flight Simulator. Ph. D. Dissertation, Harbin Institute of Technology, Harbin, China, 2013.

10. X u, C. Study on the Washout Algorithm for Stewart Platform. M. S. Thesis, Harbin Institute of Technology, Harbin, China, 2008.

11. W a ng, H., X. Ch e n. Simulating Six-DOF Motion Platform of Flight Simulator. Mechanical Science and Technology for Aerospace Engineering, Vol. 31, 2012, No 5, pp. 778-782.

12. Q i n g h a i, B. Analysis of Particle Swarm Optimization Algorithm. - Comput. Inform. Sci., Vol. 3, 2010, No 1, pp. 180-184.

13. X u, X.-B., K.-F. Zh en g, D. Li et al. New Chaos-Particle Swarm Optimization Algorithm. Journal on Communications, Vol. 33, 2012, No 1, pp. 24-30.

14. Xi a n g, Y., X.-J. M a, C. Li u et al. Estimation of Model Parameters and SOC of Lithium Batteries Based on IPSO-EKF. - ACTA ARMAMENTARII, Vol. 35, 2014, No 10, pp. 1659-1666.

15. H u, C. Research of 6-DOF Locomotive Driver Training Platform. M. S. Thesis, Southwest Jiaotong University, Chongqing, China, 2005.

16. Dag d e le n, M., G. Rey mond, A. Ke meny, M. B ordier, N. Maizi. Model-Based Predictive Motion Cueing Strategy for Vehicle Driving Simulators. - Control Engineering Practice, Vol. 17, 2009, No 9, pp. 995-1003.

17. Zh an g, Y., S. W ang, P. Philli ps, G. Ji. Binary PSO with Mutation Operator for Feature Selection Using Decision Tree Applied to Spam Detection. - Knowledge-Based Systems, 2014, No 64, pp. 22-31.

18. V a s s i l e v a, M., K. G e n o v a, V. V a s s i l e v. A Classification Based Interactive Algorithm of Multicriteria Linear Integer Programming. - Cybernetics and Information Technologies, Vol. 1, 2001, No 1, pp. 1-15.

19. L v, J., X. Y o u, S. L i u. $\alpha$-Nearness Antolony System with Adaptive Strategies and Performance Analysis. - Cybernetics and Information Technologies, Vol. 15, 2015, No 1, pp. 1-11. 